\title{
Relationship between the level of sacral resection and short-term outcomes in patients undergoing total pelvic exenteration combined with sacral resection for rectal cancer
}

Koji Komori ( $\nabla$ kkomori@aichi-cc.jp )

Aichi Cancer Center Hospital

Satoshi Tsukushi

Aichi Cancer Center Hospital

Masahiro Yoshida

Aichi Cancer Center Hospital

Takashi Kinoshita

Aichi Cancer Center Hospital

Yusuke Sato

Aichi Cancer Center Hospital

Akira Ouchi

Aichi Cancer Center Hospital

Seiji Ito

Aichi Cancer Center Hospital

Tetsuya Abe

Aichi Cancer Center Hospital

Kazunari Misawa

Aichi Cancer Center Hospital

Yuichi Ito

Aichi Cancer Center Hospital

Seiji Natsume

Aichi Cancer Center Hospital

Eiji Higaki

Aichi Cancer Center Hospital

Masataka Okuno

Aichi Cancer Center Hospital

Hironori Fujieda

Aichi Cancer Center Hospital

Shoji Kawakatsu

Aichi Cancer Center Hospital 


\section{Aina Kunitomo}

Aichi Cancer Center Hospital

\section{Satoshi Oki}

Aichi Cancer Center Hospital

\section{Shingo Maeda}

Aichi Cancer Center Hospital

\section{Takuya Nagao}

Aichi Cancer Center Hospital

\section{Tsukasa Aritake}

Aichi Cancer Center Hospital

\section{Kakeru Tawada}

Aichi Cancer Center Hospital

\section{Satoru Akaza}

Aichi Cancer Center Hospital

\section{Yasuhiro Shimizu}

Aichi Cancer Center Hospital

\section{Research Article}

Keywords: total pelvic exenteration combined with sacral resection, sacral resection, short-term outcome, curability, rectal cancer

Posted Date: March 29th, 2022

DOI: https://doi.org/10.21203/rs.3.rs-1393721/v2

License: (c) (1) This work is licensed under a Creative Commons Attribution 4.0 International License. Read Full License 


\section{Abstract \\ Background}

This retrospective study aimed to elucidate the relationships between short-term outcomes following total pelvic exenteration combined with sacral resection (TPES) and operative techniques in patients with rectal cancer.

\section{Methods}

Data regarding sex, age, body mass index, neoadjuvant therapy, location of sacral resection ("Upper" or "Lower" relative to the level between the 3rd and 4th sacral segment), operative time, bleeding, and curability (R0/R1) were collected and compared to determine their association with complications exhibiting a Clavien-Dindo grade higher than III.

\section{Results}

The complication rate was significantly higher for recurrent cancers than for primary cancers $(p=0.007)$, and for "Upper" resection than for "Lower" resection $(p=0.078)$. Significant differences were observed when complication rates for "Upper" and "Lower" resection were compared between primary and recurrent cancers $(p=0.007)$.

\section{Conclusion}

In patients with recurrent rectal cancer, "Upper" sacral resection during TPES is associated with a high complication rate, highlighting the need for careful monitoring.

\section{Introduction}

Total pelvic exenteration combined with sacral resection (TPES) is highly invasive and is associated with a high frequency of complications. Currently, minimally invasive procedures are the mainstay of colorectal surgery, ${ }^{1}$ and most forms of highly invasive surgery have been replaced by chemotherapy, especially in cases of recurrent rectal cancer. ${ }^{2,3}$ However, when the cancer is refractory to chemoradiation or abundant bleeding is observed from the tumor, TPES remains an essential technique. Despite this, the short-term outcomes of TPES (e.g., complication rates) have not been sufficiently recognized due to the scarcity of previous studies. Therefore, in this retrospective study, we aimed to elucidate the relationships between short-term TPES outcomes and operative techniques in patients with primary and recurrent rectal cancers, and to demonstrate our surgical technique for TPES. 


\section{Patients And Methods}

This retrospective study was approved by the Ethics Committee of our institution (2020-1-096) and conformed to the provisions of the Declaration of Helsinki. The study included 20 patients who underwent TPES for primary and recurrent rectal cancers in the Department of Gastroenterological Surgery at Aichi Cancer Center Hospital (Nagoya, Japan) between January 2004 and April 2020. Among the 20 included cases, seven involved primary lesions, while 13 involved recurrent lesions. All patients were confirmed to have adenocarcinoma.

"Post TPES" was defined as preservation of the bladder with removal of the other pelvic organs (i.e., rectum, uterine adnexa, and a portion of the sacrum). In contrast, "Total TPES" was defined as removal of all pelvic organs, including the bladder, rectum, uterine adnexa, prostate, and a portion of the sacrum.

We conducted a review of the hospital records to obtain the following clinicopathological information for each patient: sex (male or female), age ( $\geq 60$ or $<60$ years, median: 60 years), body mass index (BMl, $\geq 23$ or $<23 \mathrm{~kg} / \mathrm{m}^{2}$, median: $23 \mathrm{~kg} / \mathrm{m}^{2}$ ), neoadjuvant therapy (presence or absence), surgical procedure (Post TPES or Total TPES), location of sacral resection ("Upper", defined as resection above the level between the 3rd and 4th sacral segment, or "Lower", defined as resection below the level between the 3rd and 4th sacral segment), operative time ( $\geq 750$ or $<750$ min, median: $750 \mathrm{~min}$ ), operative bleeding ( $\geq 3,000$ or $<3,000 \mathrm{ml}$, median: 3,000 ml), complications with a Clavien-Dindo grade higher than III4 (presence or absence), and curability (R0 or R1).

We retrospectively evaluated short-term outcomes in terms of these clinicopathologic factors as well as the efficacy of our TPES technique.

\section{Statistical analysis}

All data are expressed as the mean \pm SD. The Fisher exact probability test, univariate logistic regression, and multivariate stepwise logistic regression were subsequently performed to identify factors that may influence clinicopathologic variables. The log-rank test was used to evaluate differences in the overall survival rate and disease-free survival rate. The level of statistical significance was set at $p<0.05$.

\section{TPES technique}

In our surgical strategy for TPES, the level of sacrectomy is confined below the 2 nd sacral segment. Sacrectomy performed at very high levels (i.e., resection above the level between the 1 st and 2nd sacral segments) is not indicated due to the risk of destabilizing the pelvis.

Our surgical strategy for TPES includes three important aspects: (1) preventing injury to the ureter, (2) ensuring an adequate surgical margin, and (3) stopping unexpected cases of massive bleeding. Notably, the surgical technique is more difficult to employ in cases of recurrent lesions than in cases of primary lesions. 


\section{Preventing injury to the ureter}

Adhesion is more severe in patients with recurrent lesions than in those with primary lesions, and severe adhesion may precipitate dislocation of the pelvic organs. Notably, the ureters are often dislocated in recurrent cases, and it is difficult to recognize some organs, especially the ureter itself. Therefore, we devised the following approach. As it is critical to preserve the length of the bilateral ureters as much as possible, our technique involves catheterization of double J-stents into the ureters preoperatively, which facilitates recognition of the ureters via palpation during the operation. ${ }^{5}$

\section{Ensuring an adequate surgical margin}

Severe adhesion can make it difficult to differentiate tumor lesions from normal organs, especially during sacral resection. However, it is of paramount importance to ensure an adequate operative field around the sacrum. Colon resection, total mesorectal excision, and central lymph node dissection are then performed, following which the Santorini venous plexus is ligated using the "Bunching technique." The sacral incision line is marked at least $1 \mathrm{~cm}$ apart from the tumor using stunning Kirschner wires. The tip of the Kirschner wires penetrate the posterior gluteal skin. Subsequently, the location of the Kirschner wire in terms of sacral level is determined via roentgenography (Figure 1).

Next, the patient is transferred to the prone position, and the surgical team confirms that the tips of the Kirschner wires protrude through the gluteal skin and sacrum (Figure 2). Incisions are then made at cutaneous sites located more cephalad than the extrusions of the Kirschner wires, and the surgical team ensures that the tips of the wires protrude through the sacrum (Figure 3). This method is used to ensure an adequate surgical margin, following which the bilateral sacrotuberous ligament and sacrospinous ligament are incised (Figure 4). Extrusion of the Kirschner wires is reconfirmed, the cephalad region of the sacrum is resected using a luer or chisel, and the tumor is removed (Figure 5). Thereafter, the bilateral ureters are anastomosed into the ileum and ileal conduit, and a colostomy is created.

\section{Stopping unexpected cases of massive bleeding}

Normally, energy devices such as LigaSure ${ }^{T M}$ are used to stop bleeding. However, in cases of recurrence, massive unexpected bleeding is difficult to stop using such devices because of the fragility of the vessel walls due to severe adhesion. Therefore, in our technique, the bleeding point is compressed using gauze balls (Tsupperu), and transfixation suturing is performed using an absorbable synthetic monofilament suture $\left(\right.$ PDS $\left.^{\circledR}\right)$ (Figure 6).

\section{Results}

Table 1 shows the relationships between clinical prognostic findings and the presence or absence of complications with Clavien-Dindo grades higher than III. Complications with grades higher than III were significantly more frequent in patients with recurrent rectal cancers than in patients with primary rectal cancers $(p=0.007)$. The frequency of such complications was also significantly higher for resection 
performed in the "Upper" sacral region than for resection performed in the "Lower" sacral region $(\mathrm{p}=$ 0.078).

Table 1

Relationships between clinical prognostic findings and the presence or absence of complications with Clavien-Dindo grades higher than III

\begin{tabular}{|c|c|c|c|c|}
\hline & & Positive & Negative & p \\
\hline \multirow[t]{2}{*}{ Sex } & Male & $9(44.4 \%)$ & $5(55.6 \%)$ & \multirow[t]{2}{*}{0.202} \\
\hline & Female & $2(63.6 \%)$ & $4(36.4 \%)$ & \\
\hline \multirow[t]{2}{*}{ Age (years) } & $\geq 60$ & $4(44.4 \%)$ & $5(55.6 \%)$ & \multirow[t]{2}{*}{0.391} \\
\hline & $<60$ & $7(63.6 \%)$ & $4(36.4 \%)$ & \\
\hline \multirow[t]{2}{*}{ BMI $\left(k g / m^{2}\right)$} & $\geq 23$ & $5(71.4 \%)$ & $2(28.6 \%)$ & \multirow[t]{2}{*}{0.279} \\
\hline & $<23$ & $6(46.2 \%)$ & $7(53.8 \%)$ & \\
\hline \multirow[t]{2}{*}{ Lesion group } & Primary & $1(14.3 \%)$ & $6(85.7 \%)$ & \multirow[t]{2}{*}{0.007} \\
\hline & Recurrent & $10(76.9 \%)$ & $3(23.1 \%)$ & \\
\hline \multirow[t]{2}{*}{ Neoadjuvant therapy } & Positive & $6(66.7 \%)$ & $3(33.3 \%)$ & \multirow[t]{2}{*}{0.343} \\
\hline & Negative & $5(45.5 \%)$ & $6(54.5 \%)$ & \\
\hline \multirow[t]{2}{*}{ Location of sacral resection } & Upper & $8(72.7 \%)$ & $3(27.3 \%)$ & \multirow[t]{2}{*}{0.078} \\
\hline & Lower & $3(33.3 \%)$ & $6(66.7 \%)$ & \\
\hline \multirow[t]{2}{*}{ Surgical procedure } & Post TPES & $2(18.2 \%)$ & 9 (81.8\%) & \multirow[t]{2}{*}{0.620} \\
\hline & Total TPES & $9(75.0 \%)$ & $3(25.0 \%)$ & \\
\hline \multirow[t]{2}{*}{ Operative time (min) } & $\geq 750$ & $4(40.0 \%)$ & $6(60.0 \%)$ & \multirow[t]{2}{*}{0.178} \\
\hline & $<750$ & $7(70.0 \%)$ & $3(30.0 \%)$ & \\
\hline \multirow[t]{2}{*}{ Operative bleeding (ml) } & $\geq 3000$ & $6(66.7 \%)$ & $3(33.3 \%)$ & \multirow[t]{2}{*}{0.343} \\
\hline & $<3000$ & $5(45.5 \%)$ & $6(54.5 \%)$ & \\
\hline \multirow[t]{2}{*}{ Curability } & R0 & $9(50.0 \%)$ & $9(50.0 \%)$ & \multirow[t]{2}{*}{0.178} \\
\hline & $\mathrm{R} 1$ & $2(100.0 \%)$ & $0(0.0 \%)$ & \\
\hline
\end{tabular}

Table 2 shows the relationships for lesion type (primary vs. recurrent) and the location of sacral resection ("Upper" vs. "Lower") in terms of the frequency of complications with Clavien-Dindo grades higher than III. No patients with primary lesions who underwent "Upper" sacral resection experienced complications 
with a Clavien-Dindo grade over III $(p=0.007)$. In contrast, all patients with recurrent rectal cancer who underwent "Upper" sacral resection had such complications (100.0\%).

Table 2

Relationship of lesion type (primary vs. recurrent) and location of sacral resection (Upper vs. Lower) to the frequency of complications

\begin{tabular}{|lllll|}
\hline Lesion group & Location of sacral resection & Positive & Negative & p \\
\hline Primary $(n=7)$ & Upper $(n=3)$ & $0(0.0 \%)$ & $3(100.0 \%)$ & 0.007 \\
\cline { 2 - 4 } & Lower $(n=4)$ & $1(25.0 \%)$ & $3(75.0 \%)$ & \\
\cline { 1 - 3 } Recurrent $(n=13)$ & Upper $(n=8)$ & $8(100.0 \%)$ & $0(0.0 \%)$ & \\
\cline { 2 - 4 } & Lower $(n=5)$ & $2(40.0 \%)$ & $3(60.0 \%)$ & \\
\hline
\end{tabular}

\section{Discussion}

Recent advancements in minimally invasive surgery have led to decreases in reports regarding highly invasive procedures with long surgical times, 1 and few studies overall have reported findings for TPES. Neoadjuvant chemotherapy is now common for primary rectal cancers, while chemotherapy is common for cases of recurrent rectal cancer. ${ }^{2,3}$ The National Comprehensive Cancer Network (NCCN) Clinical Practice Guidelines in Oncology ${ }^{\mathrm{TM}}$ recommend preoperative chemoradiotherapy for patients with advanced rectal cancer. However, the efficacy of preoperative chemotherapy (without radiation) for rectal cancer without a high risk of local recurrence has not been established, ${ }^{6}$ and such treatment is not recommended. In Japan, the 2019 Guidelines for the Treatment of Colorectal Cancer published by the Japanese Society for Cancer of the Colon and Rectum recommend that resection should be strongly considered when recurrence is observed in a single organ and complete surgical resection of the recurrent tumor is possible, and complete resection is recommended. ${ }^{7}$ Thus, despite the low number of cases, TPES remains an important surgical technique. Additionally, Waters et al. reviewed reports published over a 30-year period, noting a gradual increase in the frequency of papers concerning TPES. ${ }^{8}$ The authors classified the reports into three categories based on era: 1988-2004, 2005-2010, and 2011-2018. The frequency of TPES increased over time, with rates of $16 \%$ (30 of 193 cases) in $1988-2004,11 \%$ (24 of 212 cases) in $2005-2010$, and $23 \%$ (60 of 265 cases) in $2011-2018$. This report further highlights the need for surgeons to learn TPES techniques in the foreseeable future.

Few studies have investigated the relationship between differences in the location of sacral resection and postoperative complications. Milne et al. compared outcomes between patients undergoing high and low sacrectomies, ${ }^{9}$ reporting comparable rates of R0 resection for the two groups ( $76 \%$ for high sacrectomy vs. $71 \%$ for low sacrectomy). Although this is nearly consistent with our results, the curability rate in our study was higher than that reported by Milne et al. This may be due to our technique, which requires surgeons to ensure an adequate surgical margin apart from the tumor using stunning Kirschner wires. 
Given that this technique is likely to be very effective based on our findings, we advocate for its incorporation into surgical training programs.

Further, few studies have examined complications based on the location of sacral resection. Milne et al. reported that high sacrectomies are not associated with a higher rate of major complications ( $43 \%$ for high sacrectomies vs. $36 \%$ for low sacrectomies, $P=0.612) .{ }^{9}$ This is in contrast to the $72.7 \%$ complication rate for high sacrectomies observed in the current study, although the rate was similar for low sacrectomies (i.e., $33.3 \%$ in our study). The reasons for this disparity remain to be elucidated. Moriya et al. also reported low complication rates following sacral resection when locations were classified into the following five groups: "S2 inferior margin", "S3 superior margin", "S3 inferior margin", "S4 superior margin", "S4 inferior margin". ${ }^{10}$ The "S2 inferior margin" and "S3 superior margin" categories were comparable to the "Upper" category used in our study, while the "S3 inferior margin", "S4 superior margin", and "S4 inferior margin" categories were comparable to the "Lower" category used in our study. Although the degree of complications could not be completely reconciled between Moriya et al. and the current study, the complication rate for the "S2 inferior margin" and "S3 superior margin" groups was $56.2 \%$ $(18 / 32)$, which is lower than the rate of $72.7 \%(8 / 11)$ observed in our study. On the other hand, the complication rate for the "S3 inferior margin", "S4 superior margin", and "S4 inferior margin" group was $52.0 \%(13 / 25)$, which is higher than the rate of $33.3 \%(3 / 9)$ observed in our study.

Our study also has some limitations. As the current study included both patients with primary and recurrent rectal cancers, we faced great difficulty in demonstrating the prognosis following TPES.

\section{Conclusions}

Our findings indicate that patients who undergo "Upper" resection (i.e., resection above the level between the 3rd and 4th sacral segment) during TPES exhibit a high frequency of operative complications. Thus, careful monitoring and management are required both during and after the operation.

\section{Declarations}

Acknowledgements: Not applicable.

Availability of data and materials: The datasets used and/or analyzed during the current study are available from the corresponding author on reasonable request.

Compliance with ethical standards: All procedures performed in studies involving human participants were in accordance with the ethical standards of the institutional and/or national research committee and with the 1964 Helsinki Declaration and its later amendments or comparable ethical standards. For this type of study, formal consent is not required.

Ethics approval and consent to participate: This study was approved by the ethics committee of the Ethics Committee of Aichi Cancer Center, Chikusa-ku Nagoya, Japan (2020-1-096), and a written informed 
consent was obtained from all the participants.

Consent for publication: Not applicable.

Funding: The authors declare no funding.

Conflicts of interest/Competing interests to competing interest: The authors declare that they have no conflicts of interest / competing interests to competing interest.

Author contributions: KK contributed to the conception, analysis and interpretation of data, preparation of manuscript, the design, acquisition of data, the critical review, and feedback. TK, YS, AO contributed to the design, acquisition of data, the critical review, and feedback. SI, TA, KM, YI, SN, EH, MO, HF, KS, AK, SO, $\mathrm{SM}, \mathrm{TN}, \mathrm{TA}, \mathrm{KT}, \mathrm{SA}$, and YS contributed to the design, acquisition of data, the critical review, and feedback. All authors read and approved the final manuscript.

\section{References}

1. Yamaguchi T, Konishi T, Kinugasa Y, et al. Laparoscopic Versus Open Lateral Lymph Node Dissection for Locally Advanced Low Rectal Cancer: A Subgroup Analysis of a Large Multicenter Cohort Study in Japan. Dis Colon Rectum 2017;60:954-64.

2. Doi H, Yokoyama H, Beppu N, et al. Neoadjuvant Modified Short-Course Radiotherapy Followed by Delayed Surgery for Locally Advanced Rectal Cancer. Cancers 2021;13.

3. Kadota $\mathrm{T}$, Tsukada $\mathrm{Y}$, Ito $\mathrm{M}$, et al. A phase III randomized controlled trial comparing surgery plus adjuvant chemotherapy with preoperative chemoradiotherapy followed by surgery plus adjuvant chemotherapy for locally recurrent rectal cancer: Japan Clinical Oncology Group study JCOG1801 (RC-SURVIVE study). Japanese journal of clinical oncology 2020;50:953-7.

4. Dindo D, Demartines N, Clavien PA. Classification of surgical complications: a new proposal with evaluation in a cohort of 6336 patients and results of a survey. Ann Surg 2004;240:205-13.

5. Komori K, Suda H, Kinoshita T, Sato Y, Ouchi A, Shimizu Y. Resection of the external iliac artery with axillofemoral bypass for local recurrence of rectal cancer. Techniques in coloproctology 2021.

6. Benson AB, Venook AP, Al-Hawary MM, et al. Colon Cancer, Version 2.2021, NCCN Clinical Practice Guidelines in Oncology. J Natl Compr Canc Netw 2021;19:329-59.

7. Hashiguchi Y, Muro K, Saito Y, et al. Japanese Society for Cancer of the Colon and Rectum (JSCCR) guidelines 2019 for the treatment of colorectal cancer. International journal of clinical oncology 2020;25:1-42.

8. Waters PS, Peacock $\mathrm{O}$, Warrier SK, et al. Evolution of pelvic exenteration surgery- resectional trends and survival outcomes over three decades. European journal of surgical oncology: the journal of the European Society of Surgical Oncology and the British Association of Surgical Oncology 2019;45:2325-33. 
9. Milne T, Solomon MJ, Lee P, Young JM, Stalley P, Harrison JD. Assessing the impact of a sacral resection on morbidity and survival after extended radical surgery for locally recurrent rectal cancer. Ann Surg 2013;258:1007-13.

10. Moriya Y, Akasu T, Fujita S, Yamamoto S. Total pelvic exenteration with distal sacrectomy for fixed recurrent rectal cancer. Surgical oncology clinics of North America 2005;14:225-38.

\section{Figures}

\section{Figure 1}
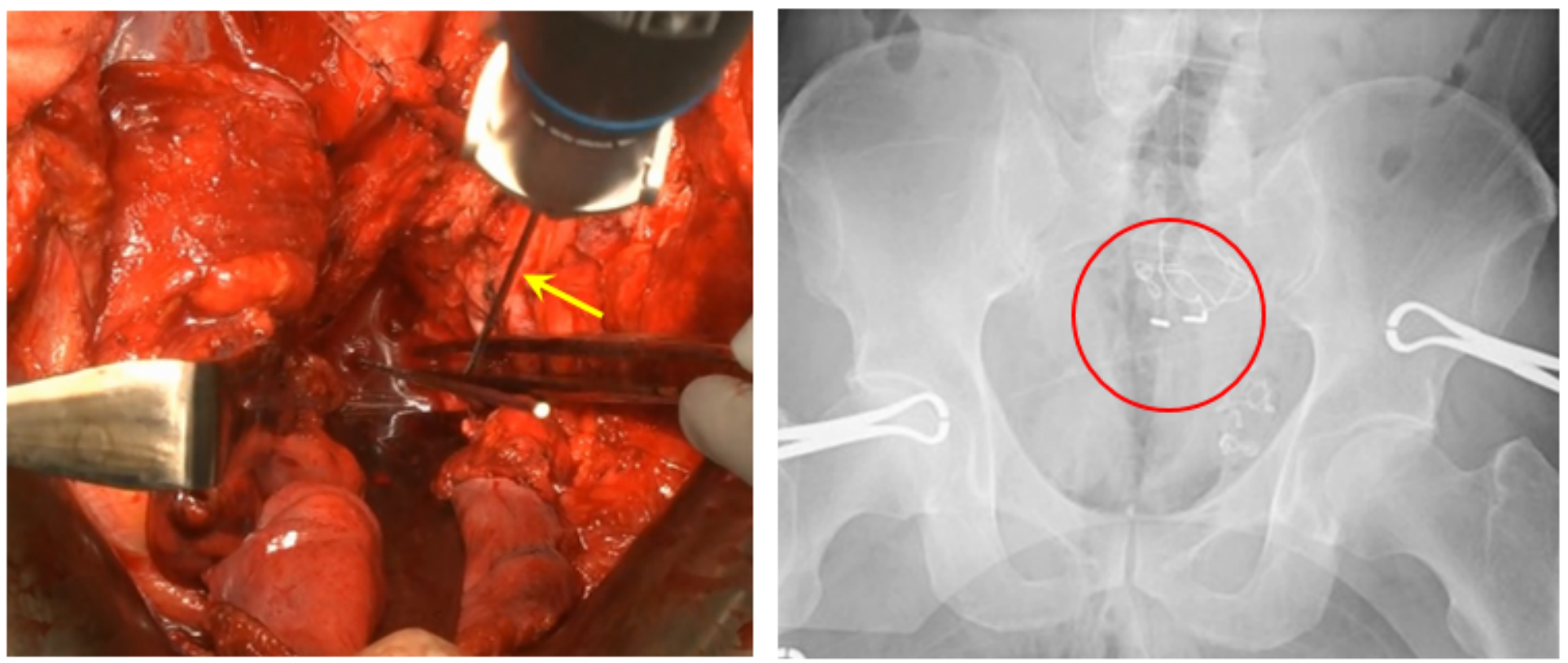

\section{Figure 1}

Ensuring an adequate surgical margin. Left side: The sacral incision line is marked at least $1 \mathrm{~cm}$ apart from the tumor using stunning Kirschner wires (yellow arrow). Right side: The site of the Kirschner wires in terms of sacral level are determined via roentgenography (red circle). 


\section{Figure 2}

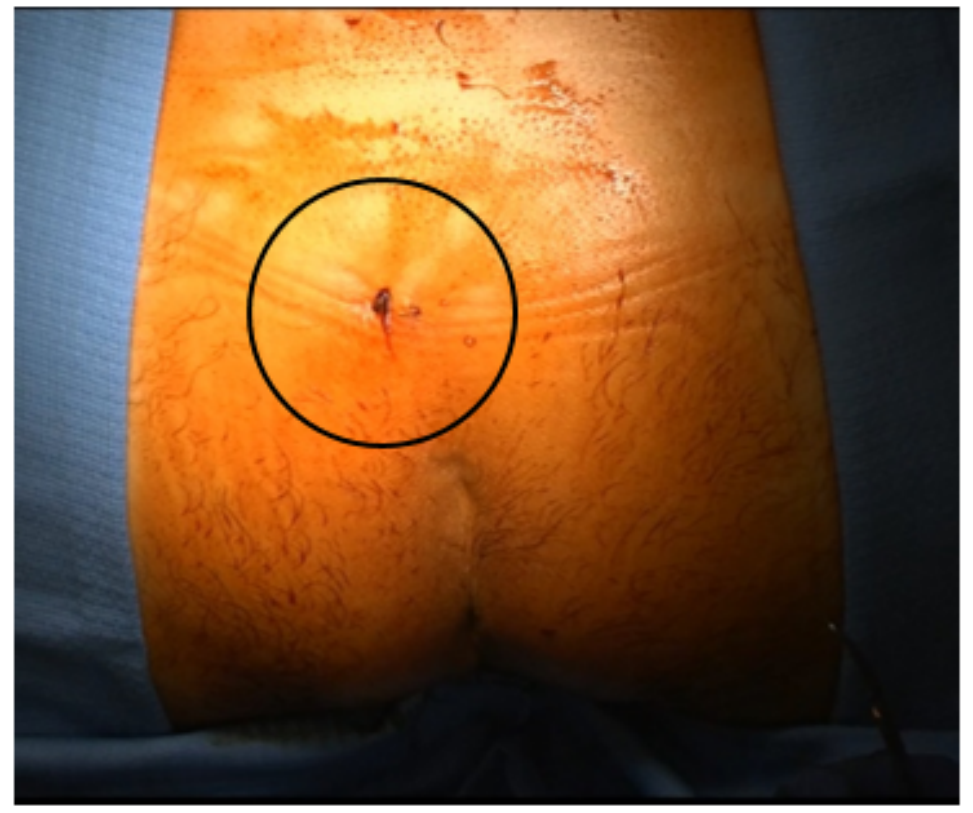

Figure 2

Transfer of the patient to the prone position. The surgical team confirms that the tips of the Kirschner wires protruded through the gluteal skin. Incisions are then made at cutaneous sites located more cephalad than the extrusions of the Kirschner wires (black circle). 


\section{Figure 3}

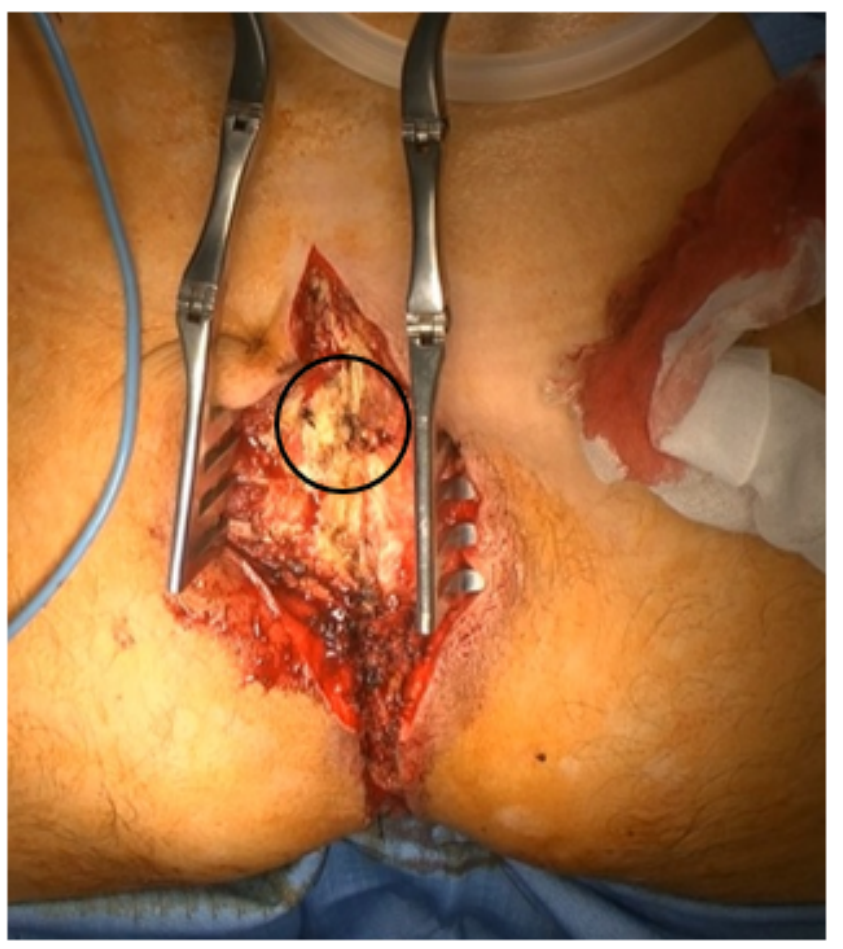

\section{Figure 3}

Confirmation that the tips of the Kirschner wires protrude through the sacrum. Protrusion sites are indicated by black arrows. 


\section{Figure 4}
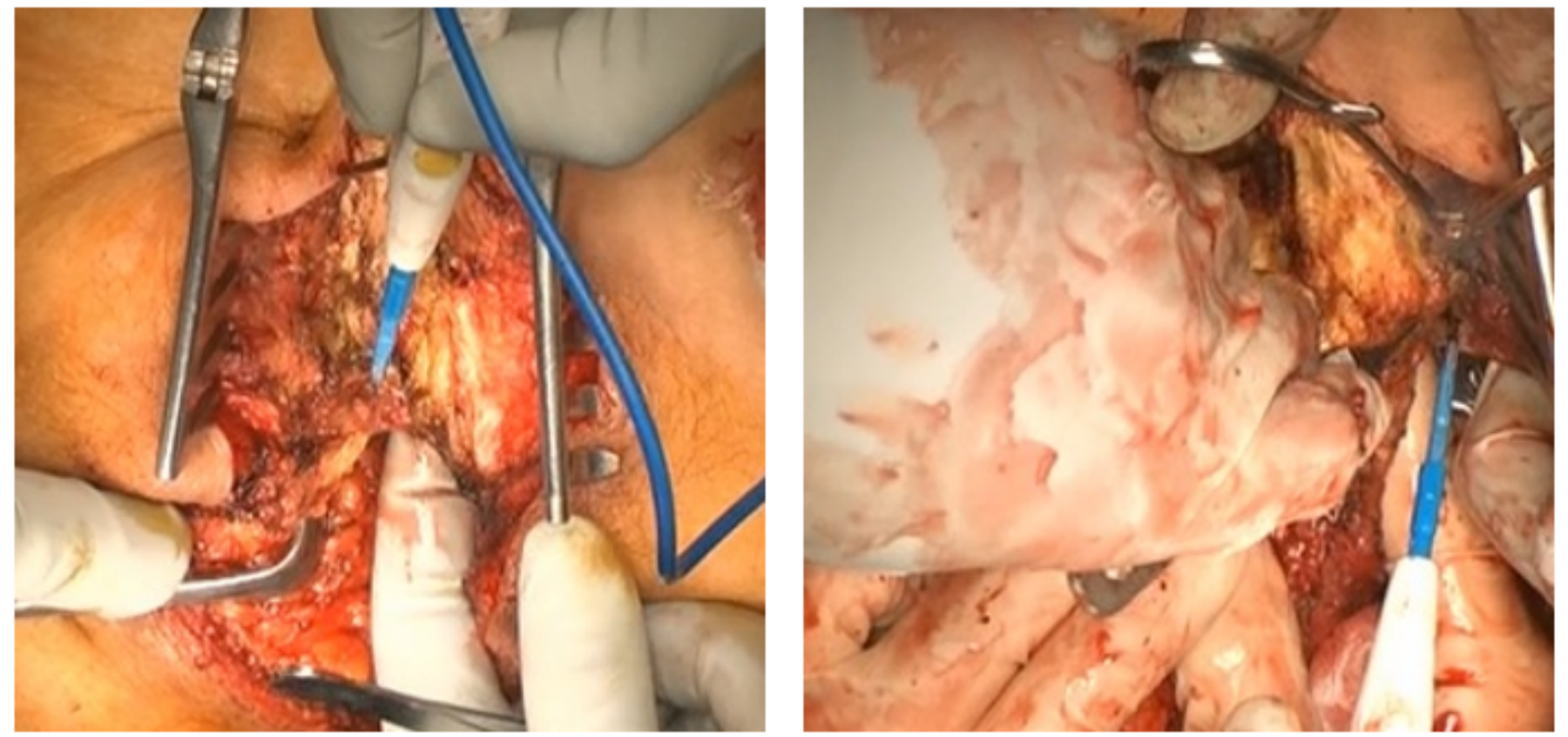

\section{Figure 4}

Incisions. Left side: Incision of the left sacrotuberous and sacrospinous ligaments. Right side: Incision of the right sacrotuberous and sacrospinous ligaments. 


\section{Figure 5}
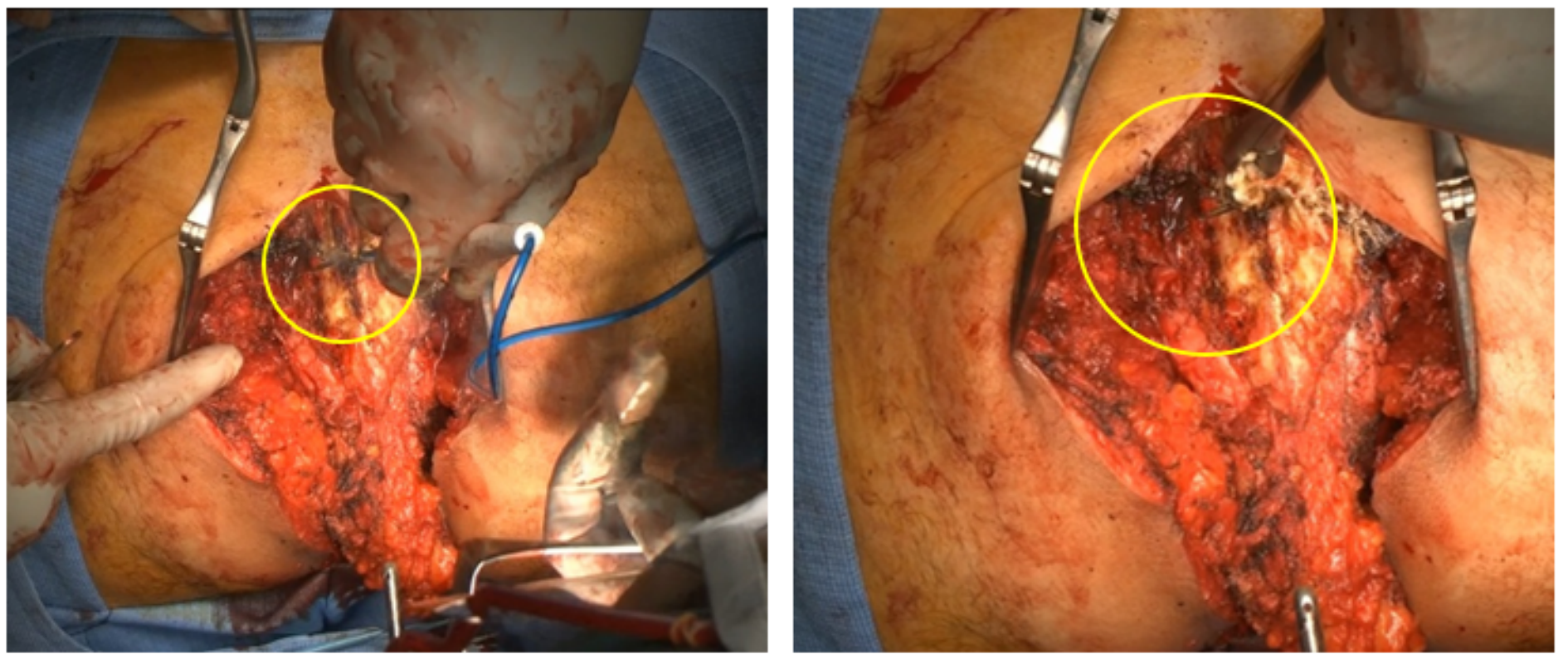

\section{Figure 5}

Resection lines. Left side: The resection line is marked more cephalad to the Kirschner wires (yellow arrow). Right side: Resection is performed between the 2 nd and 3rd sacral segment using a luer (yellow arrow). 


\section{Figure 6}
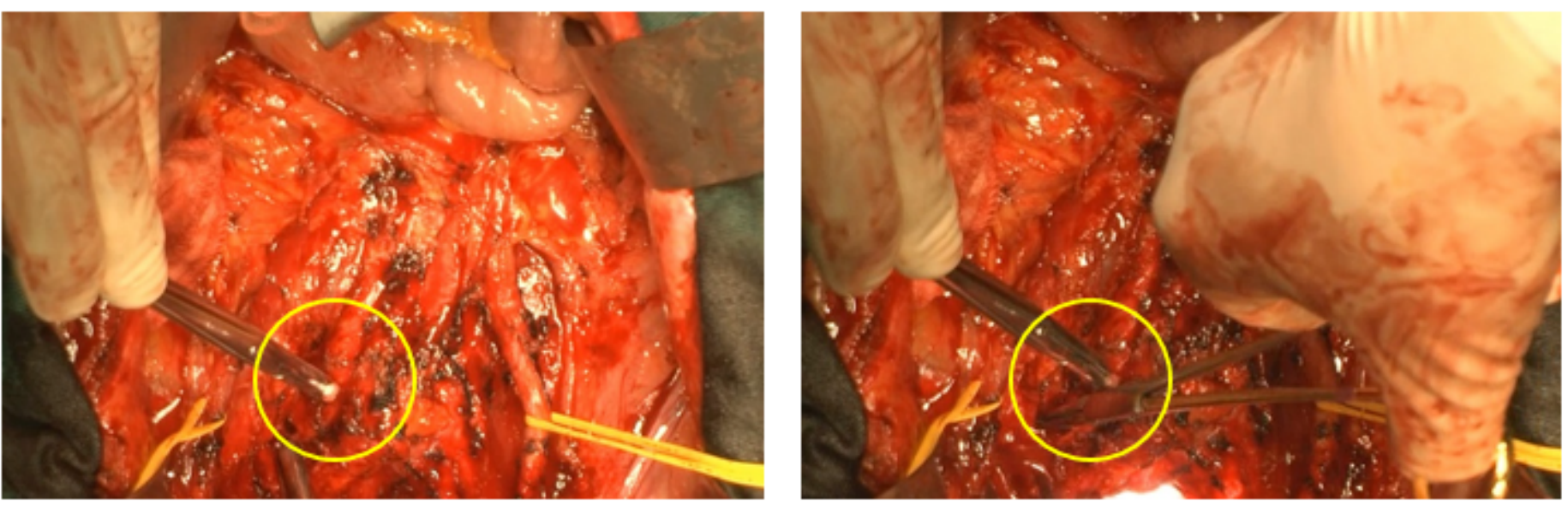

Figure 6

Addressing unexpected cases of massive bleeding. Left side: The bleeding point is compressed using gauze balls (Ts"Upper"u) (yellow arrow). Right side: Hemostasis is achieved via transfixation suturing using absorbable synthetic monofilament sutures (PDS®) (yellow arrow). 